\title{
EFECTO DE LA INMERSIÓN EN AGUA CALIENTE SOBRE LA SECRECIÓN DE LÁTEX POR LA CORONA DE GAJOS RECIÉN CONFORMADOS DE FRUTOS DE BANANO
}

\author{
Maricruz Ramírez, Marco Vinicio Sáenz ${ }^{1 / *}$, Alfonso Vargas ${ }^{* *}$ \\ Palabras clave: Banano, poscosecha, tratamiento térmico, látex. \\ Keywords: Banana, postharvest, hot water, treatment, latex.
}

Recibido: 27/11/09

\section{RESUMEN}

Con el objetivo de evaluar el efecto de la temperatura del agua y el tiempo de inmersión sobre la secreción de látex por la corona de gajos recién conformados de frutos de banano, se realizaron 2 experimentos con racimos de 12 semanas de edad (floración-cosecha) del cv. Valery (Musa AAA, subgrupo Cavendish). En el experimento 1 se evaluaron 4 temperaturas del agua: 25,35 , 45 y $55^{\circ} \mathrm{C}$ y, 4 tiempos de inmersión: 5, 10, 15 y 20 min en conjunto con un tratamiento testigo comercial. En el experimento 2 se evaluó unicamente la temperatura del agua a $45^{\circ} \mathrm{C}$ y 2 tiempos de inmersión: 10 y $20 \mathrm{~min}$. En este experimento se incluyó un tratamiento testigo comercial (frutos tomados de la línea de empaque de la finca) y otro tratamiento testigo conformado por frutos cuyos gajos fueron empacados inmediatamente después de la separación del racimo (testigo absoluto). En ambos experimentos la inmersión de los frutos en agua caliente se realizó inmediatamente después que las manos fueron seccionadas del racimo y conformadas en gajos. En ambos experimentos no hubo maduración prematura durante el periodo de simulación de transporte que fue de 15 días. En el experimento 1 los tratamientos expuestos a $55^{\circ} \mathrm{C}$, presentaron daño severo de

1 Autor para correspondencia. Correo electrónico: marco.saenz@ucr.ac.cr

Laboratorio de Tecnología Poscosecha, Centro de Investigaciones Agronómicas, Universidad de Costa Rica, San José, Costa Rica.
Aceptado: 07/03/11

\begin{abstract}
Effect of immersion in hot water of freshly-cut banana clusters on the latex secretion from the crown. Aiming to evaluate the effect of hot water treatment_both temperature and exposure time_on banana latex removal, 2 experiments were carried out using recently-harvested banana fruit (cv. Valery, Musa AAA Subgroup Cavendish) of 12 weeks of age (flower to harvest). In the first experiment, 4 water temperatures $\left(25,35,45\right.$ and $\left.55^{\circ} \mathrm{C}\right)$ and 4 immersion times (5, 10, 15 and $20 \mathrm{~min}$ ) were evaluated; a commercial control (fruit from commercial packing line) was included in the experiment. In the second experiment hot water $\left(45^{\circ} \mathrm{C}\right)$ treatments at 2 immersion times (10 and $20 \mathrm{~min}$ ) were compared with a commercial control and with an absolute control (clusters packaged just after separation from brunch). In both experiments hot water treatments were applied to recently-separated clusters (with fresh latex exudate). Hot water treatments did not induce premature onset of ripening during the transit-simulation period (15 days). In the first experiment, treatments at $55^{\circ} \mathrm{C}$ induced severe peel damage (discoloration and rotting). In general, hot water treatments did
\end{abstract}

\footnotetext{
** Depto. Investigaciones, Corporación Bananera Nacional, Costa Rica.
} 
quemadura en la cáscara. En el experimento 2 no se afectó la firmeza ( $p>0,2685)$, aunque hubo diferencias estadísticas en el color de la cáscara ( $p<0,0145)$ y en los sólidos solubles ( $\mathrm{p}>0,0001)$. El tratamiento de inmersión de $45^{\circ} \mathrm{C}$ por $10 \mathrm{~min}$ demostró que puede ser tan efectivo en la remoción del látex de la corona de gajos recién conformados de frutos de banano como el tratamiento comercial, basado en la inmersión sucesiva de los frutos en depósitos de agua circulante y la posterior aplicación de fungicidas y cicatrizantes.

\section{INTRODUCCIÓN}

El látex, tanto en la planta de banano como en el fruto cumple con la función biológica de retrasar y en ocasiones de suprimir el desarrollo de hongos y bacterias que puedan afectar partes de la planta antes de alcanzar su madurez fisiológica o perder su utilidad funcional. Químicamente se considera una suspensión coloidal de diversas organelas como lípidos, luteoides y fragmentos citoplasmáticos (Kallarackal et al. 1986) y dentro de sus constituyentes se encuentran compuestos de fenoles, terpenos, terpenolenos, agua, azúcares y proteínas entre los principales. (Baker et al. 1990).

El látex ha sido observado en todas las partes de plantas de banano in vitro, con excepción de las raíces y se encuentra a presión en los ductos laticíferos, razón por la cual, cuando se cortan uno o más ductos, su contenido es extruído (Sandoval 1989). Su concentración en la planta varía según la sección que se muestree; cuando está fresco y fluido puede lavarse, pero en pocas horas se vuelve pegajoso y finalmente se transforma en una mancha oscura sobre la piel de la fruta (Shillingford 1985). De acuerdo con Banks (1990) los frutos desflorados en el campo cerca del mediodía liberan menos látex y la severidad de la mancha es menor que aquellos donde dicha labor se efectúa en horas tempranas de la mañana. Por ello, añade el mencionado autor, es posible lograr un gran control de la mancha por not affect firmness $(\mathrm{p}>0.2685)$ but affected peel color $(\mathrm{p}<0.0145)$ and soluble solids $(\mathrm{p}<0.0001)$. Treatment at $45^{\circ} \mathrm{C}$ for $10 \mathrm{~min}$ was as effective as the commercial control (20 to $25 \mathrm{~min}$ in delatexing circulating-water tanks, followed by healing (alum) and fungicide sprays after removal from the tank), for latex removal; recoveries of latex during transport simulation were similar.

látex ocasionada por la desflora de los frutos en el campo mediante la ejecución de la labor en horas del mediodía.

La secreción de látex por la corona del gajo, una vez empacado dentro de la caja, es uno de los aspectos poscosecha que más afectan la calidad de la fruta de exportación (Miranda 1986). Su incidencia se ha asociado con factores de calidad de agua, tiempo de desleche, época del año, destino de la fruta y tipo de empaque entre otros (CORBANA 2003). Al respecto, ajustes en el $\mathrm{pH}$ del agua indican que la cantidad de látex exudado disminuyó conforme el pH decreció (CORBANA 2003).

El sistema de proceso y empaque de banano utilizado para la exportación en la actualidad, incluye el uso de depósitos con agua circulante para mantener los racimos durante periodos determinados, y favorecer el flujo y la dispersión de látex proveniente principalmente de las heridas provocadas al momento de la confección de los mismos. Al medio se le adicionan diferentes compuestos para mejorar la dispersión y evitar la formación de complejos de látex con la materia orgánica, que puede afectar la fruta o dificultar la eliminación del material (Sáenz 2004). Al respecto, Banks et al. (1988) sugieren un tiempo mínimo de permanencia del gajo en el depósito de agua circulante equivalente a 3,4 min en la época seca y de 4,1 min en la época lluviosa lo que prevendría la mancha de látex en un $99,5 \%$ de las manos. Sierra (1993) señala que 
en términos generales puede afirmarse que con 10 min de permanencia de los frutos en el agua circulante es suficiente para prevenir la mancha de látex.

Shillingford (1985) demostró que la aplicación inmediata de calor a soluciones frescas de látex logró que este coagulara y con ello una prevención del oscurecimiento causado por la oxidación. En plátano (Musa AAB) Salgado et al. (1993) menciona que la inmersión por 1 a 1,5 min a una temperatura del agua de entre 80 a $90^{\circ} \mathrm{C}$ fue superior a los restantes tratamientos térmicos evaluados. Los mencionados autores añaden que hubo una mejor respuesta del fruto a la remoción manual de la cáscara y se redujo el daño de dicha labor en la pulpa así como la mancha de látex con respecto al testigo. A su vez indican que la inmersión de los frutos en agua caliente redujo levemente la firmeza de la pulpa.

El objetivo del presente trabajo consistió en evaluar el efecto de la temperatura del agua y tiempo de inmersión sobre la secreción de látex, incidencia, severidad de enfermedades y variables poscosecha de gajos de banano.

\section{MATERIALES Y MÉTODOS}

Se realizaron 2 experimentos durante abril a junio del 2006 con frutos de banano (Musa AAA, cv. Valery) de racimos con 12 semanas (floración- cosecha) y provenientes de una finca comercial ubicada en el Caribe de Costa Rica a $23 \mathrm{msnm}$.

\section{Experimento 1: Determinación de la tolerancia de frutos de banano a la inmersión en agua a altas temperaturas}

Los racimos fueron cosechados y trasladados inmediatamente al Laboratorio Poscosecha de la Universidad de Costa Rica ubicado en la provincia de San José, cantón de Montes de Oca. Allí fueron desmanados y sus manos conformadas en gajos, los cuales se trataron de acuerdo con el tratamiento asignado.

Los tratamientos estuvieron constituidos por 4 temperaturas de agua 25, 35, $45 \mathrm{y}$ $55^{\circ} \mathrm{C}$ y 4 tiempos de inmersión: 5, 10, 15 y 20 min. Además se incluyó un testigo en el que los gajos fueron empacados sin exposición a agua caliente.

La aplicación de los tratamientos se efectuó por medio de tanques de acero de $150 \mathrm{l}$ con una cubierta aislante y 2 resistencias de 100 vatios cada uno, equipados con controles de temperatura en grados Fahrenheit con resolución de 1 grado. La temperatura se ajustó con un termómetro digital en grados centígrados. Antes y después de la aplicación de los tratamientos se midió la temperatura de pulpa y de cáscara. Al finalizar cada tratamiento se aplicó una solución de 300 mg. $1^{-1}$ de los funguicidas thiabendazole e imazalil (Lotos 40 SL, Laquinsa, Costa Rica) y Alumbre $(1 \% \mathrm{p} / \mathrm{v})$ en las coronas de los bananos incluído el tratamiento testigo.

Los gajos se empacaron en bolsas de polietileno de $0,13 \mathrm{~mm}$ de espesor con 4 orificios de $5 \mathrm{~mm}$ de diámetro en el fondo de la bolsa y luego en cajas las cuales se colocaron en una cámara de enfriamiento a $14^{\circ} \mathrm{C}$ para simular transporte durante 10 días luego del cual y antes de la exposición a etileno para inducir la maduración, se evaluaron las siguiente variables: color externo con la escala CIE L* $\mathrm{a}^{*} \mathrm{~b}^{*}$ (donde $\mathrm{L}^{*}$ es la luminosidad con valores desde 0 para negro absoluto y 100 para blanco absoluto, a* representa la variación entre verde y rojo con valores desde -50 a 0 para verdes y de 0 hasta 50 para rojos y b* la variación entre azul y amarillo con valores desde -50 a 0 para azules y de 0 a 50 para amarillos), y peso de los frutos (g), medidos respectivamente con un colorímetro Minolta ${ }^{\circledR}$ CR-300 y con una balanza AND $^{\circledR}$ GX-2000. Se evaluó la presencia de áreas oscurecidas (escaldadas) por efecto del tratamiento térmico donde la tolerancia del mercado de banano es 0 presencia de áreas con coloraciones anormales.

El diseño experimental fue factorial con 4 tratamientos de temperatura y 4 tratamientos de tiempos de inmersión. Cada tratamiento constó de 3 repeticiones y cada repetición estaba formada por 5 gajos. 


\section{Experimento 2: Evaluación de la temperatura máxima segura y el tiempo de inmersión sobre la secreción de látex por la corona en gajos de banano empacados}

Los racimos fueron cosechados y desmanados en la planta empacadora de la finca e inmediatamente sus manos seccionadas y conformadas en gajos, unidades a las que se les aplicaron los tratamientos respectivos. Los tratamientos estuvieron constituidos por una temperatura del agua: $45^{\circ} \mathrm{C}$ y 2 periodos de inmersión: 10 y $20 \mathrm{~min}$, aplicados en la planta empacadora en los mismos recipientes utilizados para el experimento 1 .

Se incluyeron 2 tratamientos adicionales: un testigo comercial conformado por cajas de banano escogidas al azar de la línea de empaque de la finca, y un testigo absoluto constituido por gajos empacados inmediatamente después de ser separados de las manos del racimo y sin tratamiento de remoción de látex.

En el testigo comercial los racimos fueron seccionados y sus manos se colocaron en agua circulante, luego se conformaron en gajos y fueron nuevamente depositados en agua circulante por 10 a 15 min para facilitar la exudación de látex por la corona. Esta fue tratada con una solución de los fungicidas tiabendazol e imazalil aplicados a una dosis de $300 \mathrm{mg} \cdot \mathrm{l}^{-1}$, un cicatrizante y astringente (alumbre al 1\%). En el testigo absoluto los frutos fueron empacados inmediatamente después de la separación de las manos del racimo y de la conformación de estas en gajos. La inmersión de los frutos en agua caliente se realizó inmediatamente después que las manos fueron seccionadas del racimo y conformadas en gajos. No se efectuó en estos, ni en el testigo absoluto, la aplicación de fungicidas ni cicatrizantes a la corona.

En cada uno de los gajos previamente pesados se colocó, alrededor de la corona del mismo, una sección de tela absorbente, $80 \%$ viscosa y $20 \%$ polipropileno de un área aproximada de $160 \mathrm{~cm}^{2}$ previamente pesados y se fijó con una banda de hule de $3 \mathrm{~mm}$ de ancho. Luego los gajos se empacaron en cajas de cartón corrugado de $18,4 \mathrm{~kg}$ netos que fueron transportadas a las instalaciones del Laboratorio de Tecnología Poscosecha en el Centro de Investigaciones Agronómicas, para ser almacenados en una cámara de enfriamiento a $14^{\circ} \mathrm{C}$ para simular transporte durante 6 días.

Una vez finalizada la simulación de transporte se registró el peso de los paños absorbentes secos y el peso de los gajos. Los tratamientos se trasladaron después a una cámara a temperatura ambiente para la aplicación de etileno. Los paños absorbentes se colocaron en un horno de convección (Fisher Scientific ${ }^{\circledR}$ ) a una temperatura de $40^{\circ} \mathrm{C}$ por un periodo de tiempo de $48 \mathrm{~h}$, hasta peso constante. Luego se determinó el peso seco del látex cuando alcanzaron temperatura ambiente con una balanza digital (AND GX-2000). Para calcular los gramos de látex exudado se utilizó la siguiente fórmula: $\mathrm{Pl}=(\mathrm{Ps}-\mathrm{Pi})-(\mathrm{Pha})$. Donde:

Ps=Peso seco final.

$\mathrm{Pi}=$ Peso inicial de los paños absorbentes colocados en las coronas de los bananos.

Pha=Peso humedad absorbida por paños absorbentes colocados dentro de las cajas.

$\mathrm{Pl}=$ Peso de látex.

Se utilizó un diseño irrestricto al azar con 4 tratamientos y 4 repeticiones. Cada repetición estuvo formada por 5 gajos.

En ambos experimentos, la aplicación de etileno fue de $100 \mu 1.1^{-1}$ por $24 \mathrm{~h}$ a $20^{\circ} \mathrm{C}$ que se efectuó en una cámara a temperatura ambiente a $20^{\circ} \mathrm{C} \mathrm{Y} 85 \%$ Humedad relativa con la ayuda de un generador catalítico (American Ripener Company Inc.) Cuando los tratamientos alcanzaron el grado 5 de maduración para banano, de acuerdo con la escala estándar de color de la cáscara de Von Loesecke (1950), se midieron las siguiente variables: color externo de la cáscara y de la pulpa( CIE L* $a^{*} b^{*}$ ), peso del fruto (g), firmeza de la pulpa (N), sólidos solubles (\%) e incidencia y severidad de enfermedades (Moho y Pudrición de corona), por medio de la metodología propuesta por Shillingford (1977) para esta última variable $0=\sin$ daño; $1=$ menos de la cuarta parte de la corona con daño; $2=$ hasta un $50 \%$ de la corona 
con daño; $3=+$ de un $50 \%$ de la corona con daño. El color externo e interno del fruto se determinó con un colorímetro electrónico (Chromameter Minolta CR-300). La firmeza de la pulpa se midió tanto en la sección basal como en la apical de cada dedo mediante un penetrómetro Mc Cormick ${ }^{\circledR}$ con cabeza convexa de 5/16”. Los sólidos solubles se determinaron por medio de un refractómetro digital (ATAGO Pallete-100) y el peso de los gajos con una balanza AND ${ }^{\circledR}$ GX-2000.

La diferencia entre peso inicial, respecto al peso final de los frutos se expresó en porcentaje como pérdida de peso. Se evaluó la presencia de áreas oscurecidas (escaldadas) por efecto del tratamiento térmico, donde la tolerancia del mercado de banano es 0 presencia de áreas de coloraciones anormales.
La exudación de látex se determinó a través del peso seco de látex atrapado en trozos de material absorbente. Dado que todo el látex producido por la fruta fue colectado, no se presentaron manchas sobre la superficie del fruto.

\section{RESULTADOS}

\section{Experimento 1: Determinación de la tolerancia de frutos de banano a la inmersión en agua a altas temperaturas}

La temperatura de la pulpa y de la cáscara de frutos de banano no alcanzaron el equilibrio con la temperatura del tratamiento térmico aplicado. El tratamiento de $55^{\circ} \mathrm{C}$ por 5 min obtuvo una temperatura final de pulpa similar a los tratamientos de $45^{\circ} \mathrm{C}$ por 15 y 20 min (Cuadro 1).

Cuadro 1. Temperatura de la pulpa de frutos de banano sometidos a diferentes temperaturas y tiempos de inmersión en agua caliente.

\begin{tabular}{|c|c|c|c|c|c|}
\hline \multicolumn{2}{|c|}{ Temperatura $\left({ }^{\circ} \mathrm{C}\right)$} & \multicolumn{4}{|c|}{ Tiempo min } \\
\hline Pulpa inicial & /Agua inicial & 5 & 10 & 15 & 20 \\
\hline & & \multicolumn{4}{|c|}{ Temperatura final pulpa $\left({ }^{\circ} \mathrm{C}\right)$} \\
\hline 20 & 25 & 22,1 & 23,2 & 23,6 & 24,7 \\
\hline 20 & 35 & 26,4 & 30,4 & 32,5 & 32,1 \\
\hline 20 & 45 & 33,8 & 38,1 & 40,9 & 40,9 \\
\hline 20 & 55 & 39,9 & 43,4 & 47,7 & 52,2 \\
\hline
\end{tabular}

Al finalizar la simulación de transporte ningún tratamiento había iniciado el proceso de maduración (Cuadro 2). En dicha etapa, la variable $\mathrm{L}^{*}$, que representa la luminosidad de la fruta, mostró diferencias significativas en relación con la temperatura $(\mathrm{p}<0,0001)$, el tiempo de exposición $(\mathrm{p}<0,0002)$ y a la interacción de ambos $(\mathrm{p}<0,0001)$ donde los frutos expuestos a $55^{\circ} \mathrm{C}$ por tiempos de exposición de 10, 15 y $20 \mathrm{~min}$ fueron más oscuros (valores de $\mathrm{L}^{*}$ menores) que el resto de los tratamientos. La variable $a^{*}$, que representa el cambio de color de verde a rojo, fue significativa con respecto a la temperatura de tratamiento $(\mathrm{p}<0,0001)$, al tiempo de inmersión $(\mathrm{p}<0,0013)$ y a la interacción de estos $(\mathrm{p}<0,0001)$, los datos muestran que los bananos expuestos a $55^{\circ} \mathrm{C}$ y a todos los tiempos de exposición fueron menos verdes. La variable $\mathrm{b}^{*}$ (cambio de azul a amarillo), presentó diferencias significativas entre los tratamientos térmicos $(\mathrm{p}<0,0001)$, los tiempos de inmersión $(\mathrm{p}<0,0083)$ y la interacción entre ambos $(\mathrm{p}<0,0007)$ lo que denota que los bananos expuestos a $55^{\circ} \mathrm{C}$ por tiempos de 10 , 15 y 20 min fueron más amarillos. En conjunto las variables de color muestran que los bananos expuestos a $55^{\circ} \mathrm{C}$ fueron más oscuros esto es, con sintomas de escaldadura por alta temperatura, menos verdes y más amarillos (sintomas de maduración) que el resto de los tratamientos evaluados. 
Cuadro 2. Color (CIE L*a*b*) de la cáscara (medias \pm error estándar) en frutos de banano (Musa AAA, cv. Valery) sometidos a diferentes temperaturas y tiempos de inmersión en agua al finalizar la simulación de transporte (Experimento 1).

\begin{tabular}{|c|c|c|c|c|}
\hline & & & Variable & \\
\hline $\mathrm{T}\left({ }^{\circ} \mathrm{C}\right)$ & Tiempo (min) & $\mathrm{L}^{*}$ & $a^{*}$ & $b^{*}$ \\
\hline \multirow{4}{*}{ Testigo } & 5 & $61,53 \pm 0,84 \mathrm{~d}$ & $-19,47 \pm 0,37 \mathrm{a}$ & $38,74 \pm 0,20 \mathrm{~d}$ \\
\hline & 10 & $61,53 \pm 0,84 \mathrm{~d}$ & $-19,47 \pm 0,37$ a & $38,74 \pm 0,20 \mathrm{~d}$ \\
\hline & 15 & $61,53 \pm 0,84 \mathrm{~d}$ & $-19,47 \pm 0,37$ a & $38,74 \pm 0,20 \mathrm{~d}$ \\
\hline & 20 & $61,53 \pm 0,84 \mathrm{~d}$ & $-19,47 \pm 0,37$ a & $38,74 \pm 0,20 \mathrm{~d}$ \\
\hline \multirow{4}{*}{25} & 5 & $58,54 \pm 0,98 \mathrm{~cd}$ & $-20,00 \pm 0,37 \mathrm{a}$ & $36,22 \pm 0,41 \mathrm{~d}$ \\
\hline & 10 & $57,48 \pm 0,26 \mathrm{bcd}$ & $-20,43 \pm 0,14 \mathrm{a}$ & $36,17 \pm 0,17 \mathrm{~cd}$ \\
\hline & 15 & $58,31 \pm 1,91 \mathrm{~cd}$ & $-20,13 \pm 0,40 \mathrm{a}$ & $35,42 \pm 0,30 \mathrm{~cd}$ \\
\hline & 20 & $59,15 \pm 1,55 \mathrm{~d}$ & $-20,13 \pm 0,30 \mathrm{a}$ & $35,93 \pm 0,47 \mathrm{~cd}$ \\
\hline \multirow{4}{*}{35} & 5 & $59,79 \pm 0,51 \mathrm{~d}$ & $-20,29 \pm 0,25 \mathrm{a}$ & $36,79 \pm 0,30 \mathrm{~d}$ \\
\hline & 10 & $56,89 \pm 1,16 \mathrm{bcd}$ & $-20,45 \pm 0,23 \mathrm{a}$ & $35,63 \pm 0,40 \mathrm{~cd}$ \\
\hline & 15 & $57,07 \pm 1,04$ bcd & $-20,55 \pm 0,12 \mathrm{a}$ & $35,75 \pm 0,35 \mathrm{~cd}$ \\
\hline & 20 & $57,39 \pm 0,76 \mathrm{bcd}$ & $-20,82 \pm 0,14 \mathrm{a}$ & $36,17 \pm 0,36 \mathrm{~cd}$ \\
\hline \multirow{4}{*}{45} & 5 & $57,42 \pm 1,19 \mathrm{bcd}$ & $-20,66 \pm 0,29$ a & $36,27 \pm 0,23 \mathrm{~d}$ \\
\hline & 10 & $57,28 \pm 0,53 \mathrm{bcd}$ & $-20,44 \pm 0,16 \mathrm{a}$ & $35,74 \pm 0,27 \mathrm{~cd}$ \\
\hline & 15 & $59,95 \pm 1,05 \mathrm{~d}$ & $-20,25 \pm 0,08 \mathrm{a}$ & $35,74 \pm 0,16 \mathrm{~cd}$ \\
\hline & 20 & $56,64 \pm 1,57 \mathrm{bcd}$ & $-20,70 \pm 0,19 \mathrm{a}$ & $35,82 \pm 0,66 \mathrm{~cd}$ \\
\hline \multirow{4}{*}{55} & 5 & $59,56 \pm 1,16 \mathrm{~d}$ & $-17,20 \pm 0,68 a b$ & $35,19 \pm 0,21 \mathrm{~cd}$ \\
\hline & 10 & $50,95 \pm 2,89 \mathrm{bc}$ & $-12,43 \pm 2,18 b$ & $28,94 \pm 2,26 \mathrm{bc}$ \\
\hline & 15 & $49,81 \pm 4,29 b$ & $-12,88 \pm 3,74 b$ & $27,72 \pm 4,56 a b$ \\
\hline & 20 & $39,27 \pm 1,50 \mathrm{a}$ & $-2,87 \pm 1,53 \mathrm{c}$ & $21,09 \pm 3,37 \mathrm{a}$ \\
\hline
\end{tabular}

Medias dentro de la misma columna con distinta letra difieren significativamente $(\mathrm{p}<0,05)$ según prueba Tukey.

Igualmente en la simulación de transporte los frutos expuestos a $55^{\circ} \mathrm{C}$ mostraron lesiones visibles en la superficie de la fruta producidas por daño térmico y que se caracterizaron por áreas de color pardo oscuro (Figura 1). Ello no ocurrió en los frutos expuestos a los demás tratamientos térmicos; con excepción del tratamiento que comprendió la inmersión de frutos por $5 \mathrm{~min}$ a $55^{\circ} \mathrm{C}$, los demás tratamientos con esa temperatura del agua fueron descartados. El tratamiento de $55^{\circ} \mathrm{C}$ por 5 min se conservó para dar seguimiento visual al desarrollo de su maduración.

La pérdida de peso en el periodo comprendido entre el empaque y el grado 5 de maduración varió $(p>0,003)$ entre tratamientos, con una mayor magnitud de pérdida en aquellos con las temperaturas del agua más altas $\left(35^{\circ} \mathrm{C}\right.$ y $\left.45^{\circ} \mathrm{C}\right)$ con respecto a los de temperaturas más bajas $\left(25^{\circ} \mathrm{C}\right.$ y testigo) para 10,15 y 20 min de inmersión (Cuadro 3). 


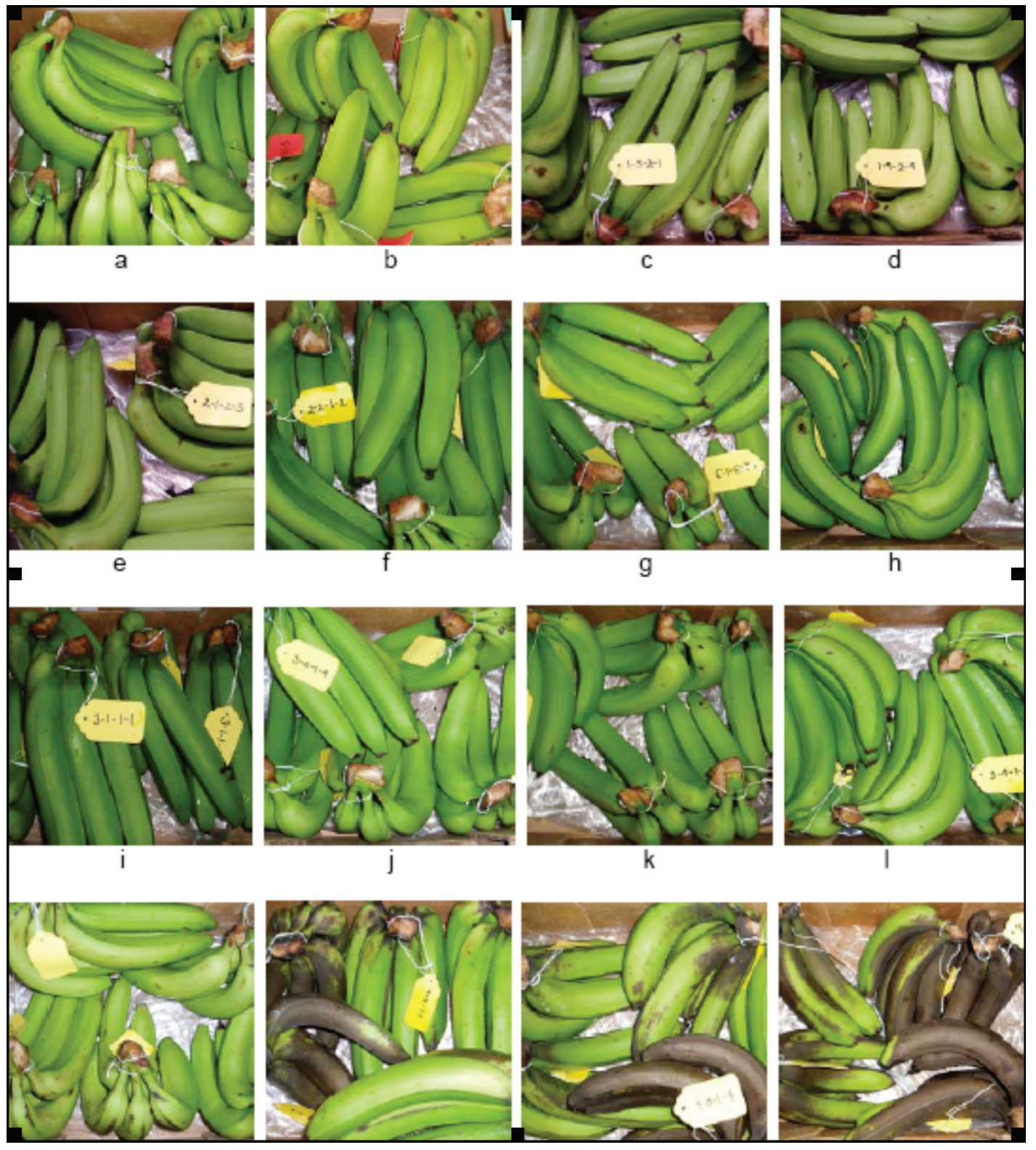

Fig. 1. Frutos de banano (Musa AAA cv. Valery) expuestos a diferentes temperaturas y tiempos de inmersión al finalizar el almacenamiento: (a) $25^{\circ} \mathrm{C}$ x $5 \mathrm{~min}$, (b) $25^{\circ} \mathrm{C}$ x $10 \mathrm{~min}$, (c) $25^{\circ} \mathrm{C}$ x $15 \mathrm{~min}$, (d) $25^{\circ} \mathrm{C} \times 20 \mathrm{~min}$, (e) $35^{\circ} \mathrm{C} \times 5 \mathrm{~min}$, (f) $35^{\circ} \mathrm{C}$ x $10 \mathrm{~min},(\mathrm{~g}) 35^{\circ} \mathrm{C}$ x $15 \mathrm{~min},(\mathrm{~h}) 35^{\circ} \mathrm{C}$ x $20 \mathrm{~min}$, (i) $45^{\circ} \mathrm{C}$ x $5 \mathrm{~min}$, (j) $45^{\circ} \mathrm{C}$ x $10 \mathrm{~min}$, (k) $45^{\circ} \mathrm{C}$ x $15 \mathrm{~min}$, (l) $45^{\circ} \mathrm{C} \mathrm{x} 20$ $\min ,(\mathrm{m}) 55^{\circ} \mathrm{C}$ x $5 \mathrm{~min},(\mathrm{n}) 55^{\circ} \mathrm{C}$ x $10 \mathrm{~min},(\tilde{\mathrm{n}}) 55^{\circ} \mathrm{C}$ x $15 \mathrm{~min},(\mathrm{o}) 55^{\circ} \mathrm{C}$ x $20 \mathrm{~min} .34$. 
Cuadro 3. Porcentaje de pérdida de peso (mediasıerror estándar) en frutos de banano (Musa AAA, cv. Valery) sometido a diferentes temperaturas y tiempos de inmersión en agua al alcanzar grado 5 de maduración (Experimento 1).

\begin{tabular}{lllll}
\hline \multicolumn{5}{c}{ Tiempo (min) } \\
\hline T $\left({ }^{\circ} \mathrm{C}\right)$ & 5 & 10 & 15 & 20 \\
Testigo & $2,14 \pm 0,07 \mathrm{cde}$ & $2,14 \pm 0,07 \mathrm{cde}$ & $2,14 \pm 0,07 \mathrm{cde}$ & $2,14 \pm 0,07 \mathrm{cde}$ \\
25 & $2,14 \pm 0,07 \mathrm{de}$ & $2,14 \pm 0,06 \mathrm{de}$ & $2,25 \pm 0,10 \mathrm{e}$ & $2,19 \pm 0,07 \mathrm{de}$ \\
35 & $2,09 \pm 0,05 \mathrm{bcde}$ & $1,87 \pm 0,30 \mathrm{abc}$ & $1,94 \pm 0,05 \mathrm{abcd}$ & $1,93 \pm 0,05 \mathrm{abcd}$ \\
45 & $2,02 \pm 0,10 \mathrm{abcde}$ & $1,86 \pm 0,03 \mathrm{ab}$ & $1,86 \pm 0,05 \mathrm{ab}$ & $1,80 \pm 0,05 \mathrm{a}$ \\
\hline
\end{tabular}

Medias dentro de la misma columna con distinta letra difieren significativamente $(\mathrm{p}<0,05)$ según prueba Tukey.

El color en la cáscara (Cuadro 4), para las variables $\mathrm{L}^{*} \mathrm{a}^{*} \mathrm{~b}^{*}$ en frutos con grado 5 de maduración no varió entre temperaturas $(\mathrm{p}>0,0844$, $\mathrm{p}>0,1388$ y $\mathrm{p}>0,7150$; respectivamente), los tiempos $(p>0,7869, p>0,6441$ y $p>0,9986$; respectivamente ) ni la misma interacción ( $\mathrm{p}>0,9904$, $\mathrm{p}>0,0705$ y $\mathrm{p}>0,7321$; respectivamente). El color de la pulpa (datos no mostrados) para las variables $L^{*} a^{*} b^{*}$ no presentó diferencias entre las temperaturas $(p>0,2159, p>0,3026$ y $p>0,3174$, respectivamente), tiempos $(\mathrm{p}>0,8551, \mathrm{p}>0,7067$ y $\mathrm{p}>0,5186$; respectivamente) y la interacción de ambas ( $p>0,4458, p>0,9742$ y $p>0,2513$; respectivamente).

Cuadro 4. Color (CIE L*a*b*) de la cáscara (medias \pm error estándar) en frutos de banano (Musa AAA, cv. Valery) sometido a diferentes temperaturas y tiempos de inmersión en agua al alcanzar grado 5 de maduración (Experimento 1).

\begin{tabular}{|c|c|c|c|c|}
\hline \multirow[b]{2}{*}{$\mathrm{T}\left({ }^{\circ} \mathrm{C}\right)$} & \multirow[b]{2}{*}{ Tiempo (min) } & \multicolumn{3}{|c|}{ Variable } \\
\hline & & $\mathrm{L}$ & $\mathrm{a}$ & $\mathrm{b}$ \\
\hline \multirow{4}{*}{ Testigo } & 5 & $75,57 \pm 0,39 \mathrm{a}$ & $-7,34 \pm 0,22 \mathrm{a}$ & $51,44 \pm 0,97 \mathrm{a}$ \\
\hline & 10 & $75,57 \pm 0,39$ a & $-7,34 \pm 0,22 \mathrm{a}$ & $51,44 \pm 0,97 \mathrm{a}$ \\
\hline & 15 & $75,57 \pm 0,39 \mathrm{a}$ & $-7,34 \pm 0,22 \mathrm{a}$ & $51,44 \pm 0,97 \mathrm{a}$ \\
\hline & 20 & $75,57 \pm 0,39 \mathrm{a}$ & $-7,34 \pm 0,22 \mathrm{a}$ & $51,44 \pm 0,97 \mathrm{a}$ \\
\hline \multirow{4}{*}{25} & 5 & $75,16 \pm 0,29 \mathrm{a}$ & $-7,40 \pm 0,43 \mathrm{a}$ & $50,67 \pm 1,56 a$ \\
\hline & 10 & $74,99 \pm 0,81 \mathrm{a}$ & $-8,27 \pm 0,33 \mathrm{a}$ & $50,59 \pm 1,11 \mathrm{a}$ \\
\hline & 15 & $75,02 \pm 0,78 \mathrm{a}$ & $-6,96 \pm 0,16 a$ & $51,28 \pm 1,45 \mathrm{a}$ \\
\hline & 20 & $74,68 \pm 0,94 \mathrm{a}$ & $-7,24 \pm 0,23$ a & $49,90 \pm 0,65 \mathrm{a}$ \\
\hline \multirow{4}{*}{35} & 5 & $74,81 \pm 0,49 \mathrm{a}$ & $-7,50 \pm 0,39 a$ & $49,71 \pm 1,30 \mathrm{a}$ \\
\hline & 10 & $75,49 \pm 0,46 \mathrm{a}$ & $-7,19 \pm 0,26 \mathrm{a}$ & $51,40 \pm 0,56 \mathrm{a}$ \\
\hline & 15 & $74,63 \pm 1,35 \mathrm{a}$ & $-8,49 \pm 0,83 \mathrm{a}$ & $50,75 \pm 1,21 \mathrm{a}$ \\
\hline & 20 & $74,49 \pm 1,20 \mathrm{a}$ & $-8,51 \pm 0,73 \mathrm{a}$ & $52,27 \pm 1,15 \mathrm{a}$ \\
\hline \multirow{4}{*}{45} & 5 & $75,74 \pm 0,37 \mathrm{a}$ & $-7,14 \pm 0,21 \mathrm{a}$ & $52,07 \pm 0,88$ a \\
\hline & 10 & $75,98 \pm 0,51 \mathrm{a}$ & $-7,23 \pm 0,20 \mathrm{a}$ & $50,82 \pm 0,83 a$ \\
\hline & 15 & $76,63 \pm 0,28 \mathrm{a}$ & $-7,93 \pm 0,27 \mathrm{a}$ & $50,36 \pm 0,93 a$ \\
\hline & 20 & $75,49 \pm 0,70 \mathrm{a}$ & $-7,29 \pm 0,44 \mathrm{a}$ & $50,18 \pm 0,62 \mathrm{a}$ \\
\hline
\end{tabular}

Medias dentro de la misma columna con distinta letra difieren significativamente $(\mathrm{p}<0,05)$ según prueba Tukey. 
La firmeza de la pulpa presentó diferencias entre temperaturas $(p<0,0272)$, pero no para los tiempos de inmersión ( $\mathrm{p}>0,4814)$ ni para la interacción entre ambos $(\mathrm{p}>0,8515)$, en este caso la menor firmeza de pulpa fue registrada para el testigo (8,88 Newtons) en comparación con los tratamientos de agua caliente (9,28 Newtons). Los sólidos solubles no variaron entre temperaturas $(p>0,3254)$, pero si entre tiempos de inmersión ( $p>0,0251)$ y la interacción entre ambos $(\mathrm{p}<0,0001) \sin$ embargo, las diferencias son tan pequeñas ( $0,7 \%$ de sólidos) que no serían percibidas por el consumidor.

El tratamiento testigo mostró los mayores porcentajes de incidencia (Cuadro 5) y severidad de moho en las coronas de los bananos tratados, aún con la aplicación de un tratamiento poscosecha de fungicida y alumbre que se aplicó a todos los tratamientos.

Las temperaturas del agua de $25^{\circ} \mathrm{C}, 35^{\circ} \mathrm{C}$ y $45^{\circ} \mathrm{C}$ no afectaron la maduración de los frutos de banano. Sólo el tratamiento de $55^{\circ} \mathrm{C}$ por un tiempo de inmersión de 5 min tuvo un retraso en la maduración. Los frutos de este tratamiento alcanzaron el grado 5 de maduración aproximadamente $48 \mathrm{~h}$ después y presentaron "manchas de madurez" (Figura 2). Durante las evaluaciones poscosecha del primer ensayo, ninguno de los bananos de los diferentes tratamientos presentó pudrición de la corona.

Se escogió, con base en los resultados, la temperatura de $45^{\circ} \mathrm{C}$ como la temperatura máxima de las evaluadas, que no ocasionó ningún negativo efecto sobre la apariencia y maduración de las fruta y por tanto sería la temperatura máxima segura a evaluar como método para remoción de látex.

Cuadro 5. Efecto de diferentes aplicaciones de temperatura y tiempo de inmersión en la incidencia de moho en la corona de gajos de banano en color de maduración 5 en el experimento 1 .

\begin{tabular}{ccccc}
\hline $\begin{array}{c}\text { Temperatura de } \\
\text { tratamiento }{ }^{\circ} \mathrm{C}\end{array}$ & 5 & \multicolumn{1}{c}{ Tiempo de exposición (min) } & 15 & 20 \\
\hline & 92 & 10 & Incidencia de moho en la corona (\%) & 92 \\
Testigo & 7 & 92 & 13 & 0 \\
25 & 0 & 33 & 20 & 0 \\
35 & 0 & 7 & 0 & 0 \\
45 & & 0 & 02 & 0 \\
\hline
\end{tabular}

Experimento 2: Evaluación de la temperatura máxima segura y el tiempo de inmersión sobre la secreción de látex de la corona en gajos de banano empacados

Al finalizar la simulación de transporte ningún tratamiento había iniciado el proceso de maduración ni mostraron síntomas visibles de daños por temperatura (datos no mostrados). Tampoco se observaron diferencias en pérdidas de peso. Al llegar a grado 5 de maduración el porcentaje de pérdida de peso no difirió ( $p>0,7317$ ) entre tratamientos. En dicha etapa las variables $L^{*}$ y a* presentaron diferencias $(p<0,0145$ y $\mathrm{p}>0,0247$; respectivamente) entre el testigo absoluto y el tratamiento de $45^{\circ} \mathrm{C}$ y $10 \mathrm{~min}$ para el primer caso y entre el testigo absoluto y los tratamientos de $45^{\circ} \mathrm{C}$ a 10 y 20 min en el segundo caso. La variable $b^{*}$ no difirió ( $\left.p>0,3304\right)$ entre tratamientos (Cuadro 6). Los gajos de banano expuestos a los diferentes tratamientos térmicos y evaluados en grado 5 de maduración mostraron menos color verde residual (valores más cercanos a 0) que el testigo comercial y el testigo absoluto lo que sugiere tendencia a maduración más uniforme.

La firmeza de la pulpa en frutos con grado 5 de maduración (datos no mostrados) no difirió $(p>0,2685)$ entre tratamientos. El porcentaje de sólidos solubles difirió entre los tratamientos térmicos de $45^{\circ} \mathrm{C}$ por 10 y 20 min (21,32 y 21,70 Brix respectivamente) y los 


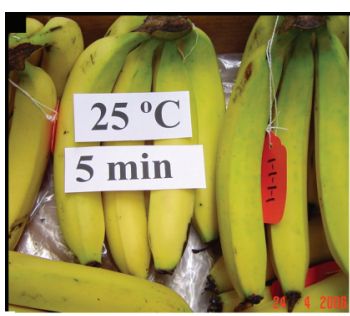

a

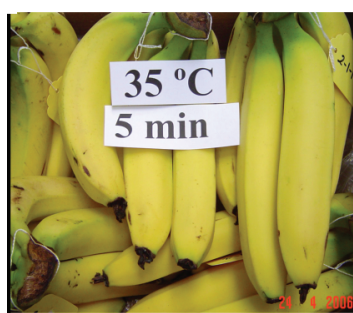

e

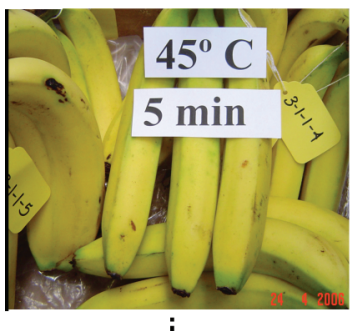

i

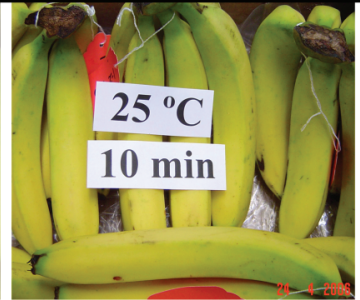

b

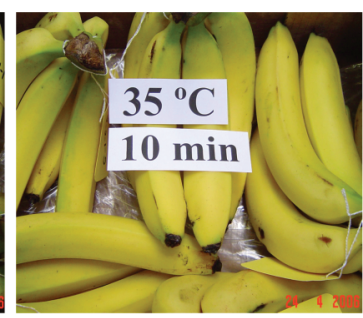

f

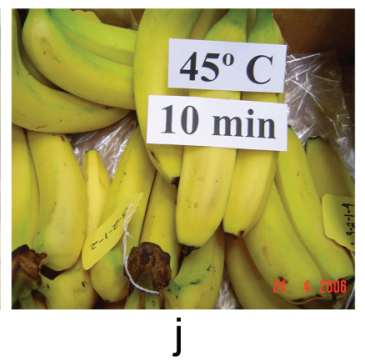

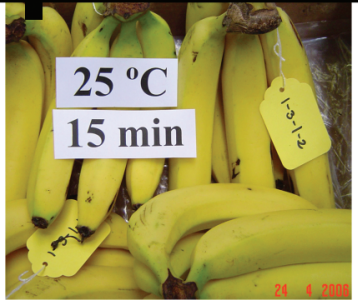

C

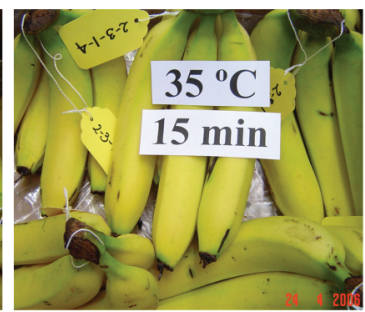

g

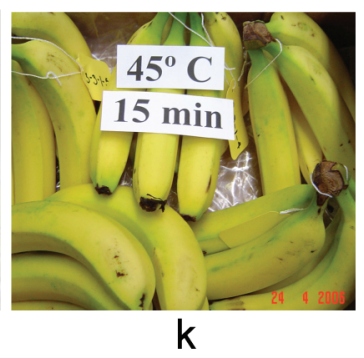

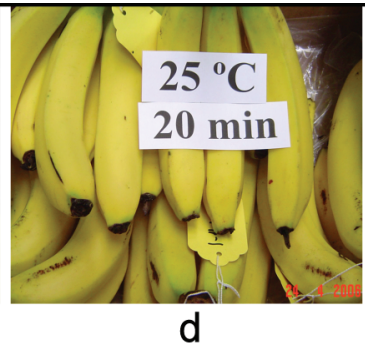

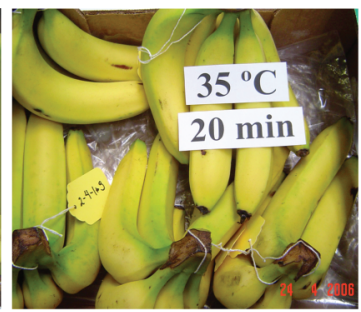

$\mathrm{h}$

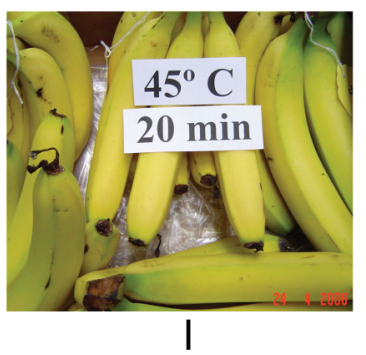

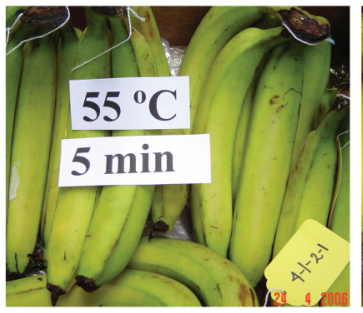

$\mathrm{m}$

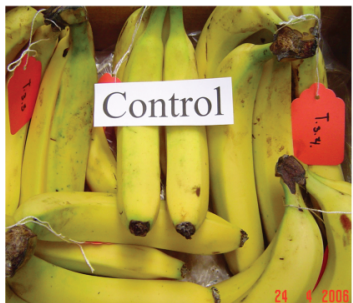

n

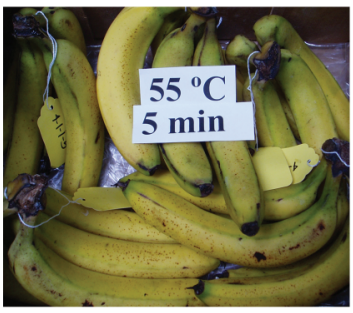

$\tilde{n}$

Fig. 2. Frutos de banano (Musa AAA cv. Valery) expuestos a diferentes temperaturas y tiempos de inmersión al grado 5 de maduración según la escala de Von Loesecke: (a) $25^{\circ} \mathrm{C}$ x $5 \mathrm{~min}$, (b) $25^{\circ} \mathrm{C}$ x $10 \mathrm{~min}$, (c) $25^{\circ} \mathrm{C}$ x $15 \mathrm{~min}$, (d) $25^{\circ} \mathrm{C}$ x 20 min, (e) $35^{\circ} \mathrm{C}$ x $5 \mathrm{~min}$, (f) $35^{\circ} \mathrm{C}$ x $10 \mathrm{~min}$, (g) $35^{\circ} \mathrm{C}$ x $15 \mathrm{~min}$, (h) $35^{\circ} \mathrm{C} \times 20 \mathrm{~min}$, (i) $45^{\circ} \mathrm{C} \mathrm{x} 5 \mathrm{~min}$, (j) $45^{\circ} \mathrm{C} \mathrm{x} 10 \mathrm{~min}$, (k)

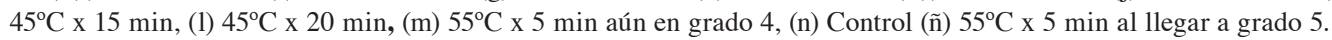

testigos absoluto (21,27 Brix) y comercial $(22,65$ Brix) $(p<0,0001)$ pero al igual que en el experimento 1 la diferencia es de entre 0,95 y $1,38 \%$ de sólidos solubles (Brix).
El peso seco (g) de látex exudado varió $(\mathrm{p}<0,0001)$ entre los tratamientos (Cuadro 7). El testigo absoluto presentó el mayor valor, dado que no recibió proceso alguno para la eliminación 
Cuadro 6. Color (CIE L*a*b*) de la cáscara (mediasıerror estándar) en frutos de banano (Musa AAA, cv. Valery) sometido a diferentes temperaturas y tiempos de inmersión en agua después de la simulación de transporte en el experimento 2.

\begin{tabular}{cccc}
\hline & & Variable & \\
Tratamiento & $\mathrm{L}^{*}$ & $\mathrm{a}^{*}$ & $\mathrm{~b}^{*}$ \\
\hline Testigo Comercial & $74,34 \pm 0,35 \mathrm{ab}$ & $-3,91 \pm 0,13 \mathrm{ab}$ & $47,71 \pm 0,80 \mathrm{a}$ \\
Testigo Absoluto & $73,47 \pm 0,30 \mathrm{a}$ & $-4,23 \pm 0,22 \mathrm{a}$ & $48,13 \pm 0,60 \mathrm{a}$ \\
$45^{\circ} \mathrm{C} \times 10 \mathrm{~min}$ & $74,91 \pm 0,28 \mathrm{~b}$ & $-3,79 \pm 0,09 \mathrm{~b}$ & $49,04 \pm 0,78 \mathrm{a}$ \\
$45^{\circ} \mathrm{C} \times 20 \mathrm{~min}$ & $74,21 \pm 0,29 \mathrm{ab}$ & $-3,60 \pm 0,11 \mathrm{~b}$ & $47,06 \pm 0,88 \mathrm{a}$ \\
\hline
\end{tabular}

Medias dentro de la misma columna con distinta letra difieren significativamente $(\mathrm{p}<0,05)$ según prueba Tukey.

Cuadro 7. Peso seco (g) de látex exudado (medias terror estándar) en frutos de banano (Musa AAA, cv. Valery) sometido a diferentes temperaturas y tiempos de inmersión en agua y almacenados después de la simulación de transporte para el experimento 2 .

\begin{tabular}{cc}
\hline Tratamientos & Peso seco $(\mathrm{g})$ de latex exudado \\
\hline Testigo Comercial & $0,021 \pm 0,004 \mathrm{a}$ \\
Testigo Absoluto & $0,343 \pm 0,016 \mathrm{~b}$ \\
$45^{\circ} \mathrm{C} \times 10 \mathrm{~min}$ & $0,024 \pm 0,004 \mathrm{a}$ \\
$45^{\circ} \mathrm{C} \times 20 \mathrm{~min}$ & $0,010 \pm 0,005 \mathrm{a}$ \\
\hline
\end{tabular}

Medias dentro de la misma columna con distinta letra difieren significativamente $(\mathrm{p}<0,05)$ según prueba Tukey.

de látex y se empacó recién seccionados los gajos. Los tratamientos térmicos, de 10 y 20 min mostraron valores estadísticamente iguales al tratamiento comercial de manejo de frutos (permanencia de $20 \mathrm{~min}$ en depósitos de agua circulante).

El principal problema fitopatológico en la corona de gajos en grado 5 de maduración fue la presencia de moho (principalmente Fusarium sp), que se presentó en los gajos expuestos a temperaturas de $45^{\circ} \mathrm{C}$, incluso un $20 \%$ de la fruta tratada por 20 min a esa temperatura presentaba un $75 \%$ de área en la corona con presencia de moho.

La pudrición de la corona (Colletotrichum sp.) y manchas severas de látex se presentaron únicamente en el testigo absoluto. Sin embargo, los gajos con tratamiento térmico presentaron una incidencia del $10 \%$ de manchas por látex leves en los tiempos de exposición de 10 y $20 \mathrm{~min}$.

\section{DISCUSIÓN}

Según Lurie (1998) la temperatura y tiempo de exposición en un tratamiento térmico, pueden aumentar o retrasar la respiración climatérica de los frutos; además de la respiración de los frutos, la salida del dióxido de carbono y vapor agua representan una pérdida de peso en el producto y por tanto una disminución de su valor comercial (Kays 1997).

Los frutos del tratamiento testigo obtuvieron una pérdida de peso menor que los frutos de los tratamientos térmicos dado que la temperatura y el tiempo de inmersión fueron factores 
generadores de estrés que aceleraron el proceso metabólico normal (Cuadro 1).

Al evaluar la calidad externa de la fruta, se identificó una tendencia a disminuir la luminosidad o brillantez de la cáscara conforme aumenta la temperatura de inmersión, desde un testigo con una luminosidad natural hasta frutos expuestos a $55^{\circ} \mathrm{C}$ con lesiones oscuras en la cáscara. Las temperaturas intermedias $\left(25^{\circ} \mathrm{C}, 35^{\circ} \mathrm{C}\right.$ y $\left.45^{\circ} \mathrm{C}\right)$ obtuvieron valores de luminosidad estadísticamente iguales al testigo.

En los tratamientos de 10, 15 y $20 \mathrm{~min}$ a $55^{\circ} \mathrm{C}$ fueron más evidentes las lesiones de la cáscara en los frutos. Este resultado concuerda con el obtenido por Reyes et al. (1998) en frutos de banano del cultivar 'Williams', los cuales presentaron lesiones en un $100 \%$ de la cáscara al aplicar tratamientos de 10-20 min de inmersión a una temperatura de $55^{\circ} \mathrm{C}$, mientras que un tratamiento de $50^{\circ} \mathrm{C}$ por $20 \mathrm{~min}$ tuvo poco efecto. Los efectos indirectos primarios del estrés causado por la aplicación de altas temperaturas pueden ser la inhibición de la síntesis de pigmentos, la formación de lesiones en la cáscara y degradación de proteínas (Kays 1997).

De acuerdo con Soto (1992), en el grado de maduración 5 se expresa el color ideal para colocar en los exhibidores de los detallistas. Los valores de $L^{*}, a^{*}$ y $b^{*}$ en la cáscara y en la pulpa reflejan que las temperaturas aplicadas por los tiempos respectivos no interfieren en el desarrollo del color normal de la pulpa ni el desarrollo normal de la luminosidad, la disminución de color verde y el aumento de color amarillo en la cáscara de los frutos de banano Cavendish.

El tratamiento de $55^{\circ} \mathrm{C}$ con un tiempo de inmersión de 5 min retrasó la maduración. Esto confirma, tal y como lo informó Lobo et al. (2000) en Dwarf Cavendish- tratados a $50^{\circ} \mathrm{C}$ por $15 \mathrm{~min}$, que los tratamientos térmicos de inmersión a altas temperaturas producen alteración de la maduración. Resultados similares obtuvo Wall (2004) al aplicar tratamientos de inmersión en agua caliente $\left(48,49\right.$ y $\left.50^{\circ} \mathrm{C}\right)$ por tiempos de 15 o 20 min en frutos de banano en los que supone se presentó una alteración de la síntesis de etileno.
En banano, la textura es un atributo compuesto por muchos factores como lo son la turgencia del agua en los tejidos y los componentes estructurales de los tejidos y células y la degradación de almidón (Seymour et al. 1993, Dadzie y Orchard 1996). Los frutos de los tratamientos térmicos mantuvieron una mejor firmeza al momento de llegar a grado 5 de maduración que el tratamiento testigo.

Se ha sugerido que los tratamientos térmicos producen un retraso en la pérdida de firmeza debido a la inhibición de la síntesis de enzimas hidrolíticas de la pared celular como Poligalacturonasa (Lazan et al. 1989 mencionado por Lurie 1998).

En banano se ha reportado que el metabolismo de carbohidratos puede ser alterado por la exposición a temperaturas elevadas, por ejemplo a temperaturas de $40^{\circ} \mathrm{C}$ la formación de sacarosa se detiene (Lizana 1976 mencionado por Seymour et al. 1993). Sin embargo en este trabajo, no se presentaron diferencias significativas en los sólidos solubles.

La temperatura de $55^{\circ} \mathrm{C}$ se descartó, por los daños provocados en la cáscara, no obstante, en banano 'Prata-Ana'; Moraes et al. (2006) afirman que un tratamiento de termoterapia de $56^{\circ} \mathrm{C}$ por 6 min produce una disminución de lesiones en la cáscara. Para la ejecución de este ensayo se utilizó el cultivar 'Valery' (Musa AAA), lo cual sugiere que es más sensible que el cultivar 'PrataAna' (Musa AAB) a los tratamientos térmicos.

Los resultados de incidencia y severidad del moho en las coronas de los bananos tratados demostraron que el tratamiento de $45^{\circ} \mathrm{C}$ fue el más efectivo como tratamiento físico para combatir el moho en la corona del banano.

A partir de los resultados del primer ensayo, se seleccionaron los tratamientos de $45^{\circ} \mathrm{C}$ por 10 y 20 min para ser aplicados en el siguiente. Se eligió una temperatura de $45^{\circ} \mathrm{C}$ al ser la mayor temperatura evaluada que no produjo daño tanto externa como internamente en los frutos de banano. La justificación para los tratamientos elegidos incluye también la ausencia de efectos negativos 
en variables como color externo, sólidos solubles, firmeza y las demás variables de calidad.

En el segundo experimento, el porcentaje de pérdida de peso de los tratamientos aplicados indicó que un tratamiento térmico de $45^{\circ} \mathrm{C}$ aún por 20 min no produce un aumento en la transpiración ni la pérdida de agua en los frutos tratados en empaques con pocas perforaciones donde el riesgo de deshidratación se minimiza.

Al finalizar la simulación de transporte, ningún tratamiento había iniciado el proceso de maduración y se puede afirmar que no se presentó aceleración del mismo. La luminosidad $\left(\mathrm{L}^{*}\right)$ del testigo absoluto obtuvo el menor valor lo cual indica que la fruta se encontraba más opaca en este momento, que se relacionó principalmente con la mancha producida por el látex segregado durante la simulación de transporte. La luminosidad $\left(\mathrm{L}^{*}\right)$ más alta fue la del tratamiento de inmersión por $10 \mathrm{~min}$, esta combinación de tiempo por temperatura favoreció la brillantez en los gajos de banano.

En general, el proceso de lavado de frutos elimina residuos de suciedad y agroquímicos presentes en las frutas, pero también elimina parte de las ceras naturales de la cáscara. También se ha descubierto que el agua caliente puede disolver parcialmente las ceras naturales y restos de estas podrían quedarse en el fruto y obstruir estructuras de intercambio gaseoso como los estomas (Shimshon 2005). Esto significaría que $10 \mathrm{~min}$ a $45^{\circ} \mathrm{C}$ no aumenta la solubilidad de las ceras naturales y posiblemente remueve partículas sólidas adheridas, que se traduce en una mayor brillantez de la cáscara de los gajos de banano.

Los valores obtenidos de firmeza indican que esta variable de calidad no se afectó por tratamientos térmicos. Los resultados obtenidos para la variable sólidos solubles, donde no se encontró diferencia estadística entre tratamientos, concuerda con investigaciones con otras musáceas (AAB) llevadas a cabo por Wall (2004), quien no encontró efecto de tratamientos de inmersión en agua caliente sobre el contenido de sólidos solubles, resultado similar al encontrado en éste trabajo.
Los valores de cantidad de látex exudado por el testigo absoluto representarían un valor cercano a la cantidad de látex exudado por un gajo típico de banano 'Valery'. La mayor exudación en el testigo absoluto se debe a que los paños absorbentes se colocaron después de seccionar la mano en el proceso de conformación del gajo. En ese momento la presión de turgencia del fruto era mayor y por tanto la secreción de látex por los canales laticíferos se efectuó con mayor facilidad. En adición, los frutos del tratamiento comercial permanecieron aproximadamente $20 \mathrm{~min}$ liberando el látex. En los tratamientos de agua caliente posiblemente se produjo un aumento de la presión interna de las células que contienen el látex (y por tanto su expulsión) y la solubilidad del mismo.

Se debe resaltar que la cantidad de látex exudada por los frutos de bananos tratados con agua caliente por $10 \mathrm{~min}$ fue similar al tratamiento comercial, lo que representaría un menor tiempo en el proceso de desleche si se aplicara comercialmente.

De acuerdo con los resultados obtenidos, para frutos de banano del cultivar 'Valery' un tratamiento de inmersión a una temperatura de $45^{\circ} \mathrm{C}$ por un tiempo mayor a los 10 min es comparable al tratamiento comercial en la disminución de la mancha de látex, además de no afectar variables poscosecha de calidad y por tanto podría considerarse como una alternativa en el manejo de látex en plantas empacadoras. Para esto se necesita profundizar en la metodología de aplicación y en tiempos menores y además estudiar con mayor detalle el efecto de los tratamientos térmicos sobre enfermedades poscosecha como la pudrición de la corona y el moho dado que los resultados obtenidos en éste trabajo no muestran un efecto positivo claro sobre dichas variables.

\section{LITERATURA CITADA}

BAKER D., KALLARACKAL J., MILBURN J. 1990. Water Relations of the Banana. II. Physicochemical Aspects of the Latex and Other Tissue Fluids. Aust. J. Plant. Physiol. 17:69-77. 
BANKS N.H. 1990. Factors affecting the severity of deflowering latex stain on banana bunches in the Windward Islands. Tropical Agriculture. 67(2):111114 .

BANKS N.H., JOSEPH M., BORTON C.A. 1988. Optimization of latex draining time in the postharvest handling of field packed Windward Islands bananas, pp. 393-398. In ***ed. Memorias VIII Reunión ACORBAT. 1987.Medellin, Colombia.

CORPORACIÓN BANANERA NACIONAL. 2003. Informe Anual. p. 96-97.

DADZIE B., ORCHARD J. 1996. Post-Harvest Criteria and Methods for Routine Screening of Banana/Plantain Hybrids. International Network for the Improvement of Banana and Plantain. Montpellier, France. 75 p.

KALLARACKAL J., GARLICK P.R., MILBURN J.A. 1986. Characterization of the structural inclusions in the latex of banana (Musa sp.). Can. J. Bot. 64:25912601.

KAYS S. 1997. Postharvest physiology of perishable plant products. Exon Press. 532 p.

LOBO M.G., POMAR M., MARRRERO A. 2000. Simulación de la cadena comercial del plátano de Canarias, pp. 215-218. In: Post-recolección de Frutos y Hortalizas. V Simposio Nacional y II Ibérico. Tenerife.

LURIE S. 1998. Review: Postharvest heat treatments. Postharv. Biol. and Technol. 14, 257-269.

MIRANDA J. 1986. Nueva tecnología en el control de las manchas de látex en bananos y equipo de empaque. Informe mensual UPEB 10(78-79):57-59.

MORAES W., ZAMBOLIM L., LIMA J.R., DO VALE F., SALOMAO L. 2006. Termoterapia e Quimioterapia de Banana 'Prata-Ana' Asociadas à Temperatura de Armazenamiento no Controle de Podridões em PósColheita. Fitopatol. Bras. 31(1), jan-fev.

REYES M., NISHIJIMA W., PAULL R. 1998. Control of crown rot in 'Santa Catarina Prata' and 'Williams' banana with hot water treatments. Postharvest Biology and Technology 14:71-75.
SÁENZ M.V. 2004. Situación internacional y perspectivas para el manejo poscosecha de fruta de banano: Un enfoque de Tecnología Comercial, pp. 161-171. In: ACORBAT publicación especial, XVI Reunión Internacional ACORBAT 2004, Oaxaca, México.

SALGADO T., FUNEZ P., MEDLICOTT A.P. 1993. Los efectos del tratamiento con agua caliente sobre el pelado del plátano, pp. 43-43. In: Programa de banano y plátano. Informe técnico 1992. Fundación hondureña de Investigación Agrícola. La Lima, Honduras.

SANDOVAL J. 1989. Estudio de la anatomía y morfología de plantas de Musa in vitro en condiciones de aclimatación y en el campo. Centro Agronómico de Investigación y Enseñanza. Turrialba, Costa Rica. $175 \mathrm{p}$.

SEYMOUR G., TAYLOR J.,TUCKER G. 1993. Biochemistry of fruit ripening. $1^{\text {st }}$ edition. Chapman \& Hall. 454 p.

SHILLINGFORD C. 1977. Control of banana fruit rot and of fungi that contaminate washing water. Trop. Sci. 19(4):197-203.

SHILLINGFORD C. 1985. Control de la mancha de látex. Informe mensual UPEB. Panamá. 9 (71):39-41.

SHIMSHON B. 2005. Environmentally Friendly Technologies for Agricultural Produce Quality. CRC Press. 534 p.

SIERRA S. 1993. Procesamiento de la fruta en la planta empacadora, pp. 573-631. In: El cultivo del banano, producción y comercio. Medellín, Colombia.

SOTO M. 1992. Banano cultivo y comercialización. San José, Costa Rica. 674 p.

VON LOESECKE H. 1950. Bananas. $2^{\text {nd }}$ edition. InterScience, New York.

WALL M. 2004. Ripening behaviour and quality of 'Brazilian' bananas following hot water inmersion to desinfest surface insects. HortScience 39(6)13491353. 